\title{
Mathematical modelling and analysis of thermometric diagnostics of electrical products
}

\author{
Danil V. Shaykhutdinov, and Konstantin M. Shirokov* \\ Platov South-Russian State Polytechnic University (NPI), Information Measuring Systems and \\ Technologies Department, 346428 Novocherkassk, Russia
}

\begin{abstract}
The actual problem is the diagnosis and monitoring of the technical condition of the machines in operation, as well as the forecasting of their technical condition. The complexity of the problem of condition controlling is that the most informative characteristics of the electrical products can not be accurately determined without the use of special measuring transducers, which considerably increases the test time and makes it difficult to carry out continuous monitoring. A promising direction in solving this problem is the use of sensorless methods. Implementing these methods is based on using as a source of information the working winding of the electrical product, the active component of the resistance of which is determined just before the measurement of the magnetic characteristic and then used as a constant value. In the process of testing, there is heating and, as a consequence, a change in the active component of the winding resistance, which causes an unacceptable errors in determining the electrical products state. In this article the mathematical model of thermal processes are developed and analysis of thermometric diagnostics of electrical products is carried out.
\end{abstract}

\section{Introduction}

The complexity of the designs of electrical products (EP), the trend of miniaturization and the reduction of material consumption make ever higher demands on the quality of the component parts $[1,2]$. The quality of the parts is determined primarily by their mechanical, electrical and magnetic properties. And it is the magnetic properties that determine the performance of this EP, and the use of parts with high magnetic properties, for example as magnetic circuits, allows to reduce the dimensions of this EP. Information on the magnetic properties of parts allows you to detect defects in the assembly stages, and identify shortcomings of other parts of the product by testing the EP assembly. Using the results of tests of blanks and products in the production process control subsystem of the EP, it is possible to increase the yield of suitable products and, thereby, significantly reduce economic costs [3-5].

No less relevant is the diagnosis and monitoring of the technical condition of the machines in operation, as well as the forecasting of their technical condition, the sorting of the output, depending on the provision of the required parameters [6].

* Corresponding author: konstantin-shirokov@mail.ru 
The complexity of the problem of controlling magnetic properties is that the most informative characteristics of the EP can not be accurately determined without the use of special measuring transducers, which considerably increases the test time and makes it difficult to carry out continuous monitoring. A promising direction in solving this problem is the use of sensorless control methods [7]. Implementing these methods of magnetic control means as a source of magnetic field use the working winding of the EP, the active component of the resistance of which is determined just before the measurement of the magnetic characteristic and then used as a constant value. In the process of testing, there is heating and, as a consequence, a change in the active component of the winding resistance, which causes an unacceptable error in determining the magnetic flux and, as a consequence, the weber-ampere characteristic of the EP.

\section{Materials and methods}

Two thermal processes lead to a change in the temperature of the winding: an increase in temperature due to heat generation during the flow of electric current along it and a change in the temperature of the winding, due to thermal exchange with the environment (air, other details of the EP) [8-9].

The resulting change in winding temperature is determined by the amount of heat received or given, in accordance with expression:

$$
d Q_{1}=c m \Delta T
$$

where $d Q_{1}$ - amount of heat received or returned; $c$ - specific heat of winding; $m$ winding mass; $\Delta T$ - variation of the temperature.

According to the Joule-Lenz law, the amount of heat received by the winding when an electric current flows through it is given by:

$$
d Q_{2}=i^{2} R\left(t^{\circ} \mathrm{C}\right) d t
$$

where $i$ - current in the winding; $R\left(t^{\circ} \mathrm{C}\right)$ - active component of winding resistance (ACWR); $d t$ - time differential.

Let's consider a particular case in which the electromagnet under test has a steady room temperature and the process of measuring the magnetic characteristic is fast enough, so that the heat exchange between the EP winding and the surrounding medium can be neglected. In this case, all the amount of heat released in the winding, due to the flow of electric current through it [10], will go to increase its temperature, that is, $d Q_{1}=d Q_{2}$, and:

$$
c m \Delta T=i^{2} R\left(t^{\circ} \mathrm{C}\right) d t .
$$

If we express from this relation the changing in temperature we will get next expression:

$$
\Delta T=\frac{i^{2} R\left(t^{\circ} \mathrm{C}\right) d t}{c m} .
$$

The current value of the active component of the winding resistance from temperature is given by:

$$
R\left(t^{\circ} \mathrm{C}\right)=R_{\mathrm{I}} \cdot(1+\alpha \Delta T)
$$

where $R_{\mathrm{I}}$ - ACWR, determined immediately before the measurement of the magnetic characteristic.

Substitute the value in this expression $\Delta T$, then:

$$
R\left(t^{\circ} \mathrm{C}\right)=R_{\mathrm{I}} \cdot\left(1+\alpha \cdot \frac{i^{2} R\left(t^{\circ} \mathrm{C}\right) d t}{\mathrm{~cm}}\right) .
$$


Expressing the current value of the active component of the winding resistance from this expression, and we will obtain:

$$
R\left(t^{\circ} \mathrm{C}\right)=\frac{c m R_{\mathrm{I}}}{c m-\alpha R_{\mathrm{I}} i^{2} d t} .
$$

Using the expression (2), it is possible to calculate the value of the active component of the winding resistance at any time when determining the magnetic characteristic of the electromagnet, using as current $i$ the value of the effective current, without taking into account the heat transfer between the winding and the environment, the change in the specific heat due to the temperature change, and The change in the active component of the winding resistance between its initial change and the current calculated value [11].

Iterative calculation avoids the last drawback and uses instantaneous current values in the calculation (also the values obtained can be used in the iterative calculation of flux linkage):

$$
R\left(t^{\circ} \mathrm{C}\right)_{k}=\frac{c m R\left(t^{\circ} \mathrm{C}\right)_{k-1}}{c m-\alpha R\left(t^{\circ} \mathrm{C}\right)_{k-1} i_{k}^{2} \cdot \Delta t},
$$

where $i_{k}$ - current value for each $k$ measurement (for $k=1 . . N$ - the number of measured points, when determining the magnetic characteristic); $\Delta t=\frac{t_{\mathrm{i}}}{k}=\left(t_{k}-t_{k-1}\right)$ - sampling step (differential of measurement time $t_{i}$ of magnetic characteristic); wherein $R\left(t^{\circ} \mathrm{C}\right)_{0}=R_{\mathrm{I}}$.

Let's analyze the expressions (2), (3) taking into account the world tendency to reduce the weight and size parameters, that is: $m \rightarrow 0$ :

$$
R\left(t^{\circ} \mathrm{C}\right)=\lim _{m \rightarrow 0} \frac{c m R_{\mathrm{I}}}{c m-\alpha R_{\mathrm{I}} i^{2} d t}=\lim _{m \rightarrow 0} \frac{\frac{c m R_{\mathrm{I}}}{c m}}{\frac{c m}{c m}-\frac{\alpha R_{\mathrm{I}} i^{2} d t}{c m}}=\lim _{m \rightarrow 0} \frac{R_{\mathrm{I}}}{1-\frac{\alpha R_{\mathrm{I}} i^{2} d t}{c} \cdot \frac{1}{m}}=\lim _{m \rightarrow 0} \frac{R_{\mathrm{I}}}{1-\frac{k}{m}},
$$

where coefficient $k=\frac{\alpha R_{\mathrm{I}} i^{2} d t}{c}>0$ is a positive constant; because tabular values of coefficients for copper $\alpha \approx 0,004>0$ and $c \approx 385>0$ always positive; any value of the active component of the resistance is always positive $\left(R_{\mathrm{I}}>0, R\left(t^{\circ} \mathrm{C}\right)_{k-1}>0\right)$; the square of the current value of the current is positive $i^{2}>0$ (the instantaneous value of the current enters into expression (3), which can take a zero value at separate intervals, then, due to the lack of heating, the active component of the resistance will not change and expression (3) will take the form: $\left.R\left(t^{\circ} \mathrm{C}\right)=\lim _{m \rightarrow 0} \frac{R\left(t^{\circ} \mathrm{C}\right)_{k-1}}{1-\frac{k}{m}}=\lim _{m \rightarrow 0} \frac{R\left(t^{\circ} \mathrm{C}\right)_{k-1}}{1-\frac{0}{0}}=R\left(t^{\circ} \mathrm{C}\right)_{k-1}\right)$; where $d t>0$ period of time, long enough to not consider that $d t \rightarrow 0$ and hence $\lim _{\substack{m \rightarrow 0 \\ d t \rightarrow 0}} \frac{\alpha R_{\mathrm{I}} i^{2}}{c} \cdot \frac{d t}{m}=\lim _{\substack{m \rightarrow 0 \\ d t \rightarrow 0}} k_{2} \cdot \frac{d t}{m}=k \cdot \frac{0}{0}$, those the uncertainty that needs to be revealed) and it is always positive. In this way, $\lim _{m \rightarrow 0} \frac{k}{m} \rightarrow+\infty$.

$$
\text { It } R\left(t^{\circ} \mathrm{C}\right)>0 \text { then } R\left(t^{\circ} \mathrm{C}\right)=\lim _{m \rightarrow 0} \frac{R_{\mathrm{I}}}{1-\frac{k}{m}}>0 \text {; those } R_{\mathrm{I}}>0 \text {, то } 1-\frac{k}{m} \geq 0 \text { or } \frac{k}{m} \leq 1 \text {. }
$$

Thus we obtain a system for which the expressions (2) and (3) make sense: 


$$
\left\{\begin{array}{l}
\lim _{m \rightarrow 0} \frac{k}{m} \rightarrow+\infty \\
\frac{k}{m} \leq 1
\end{array}\right.
$$

Thus $\frac{k}{m}=1$ is the maximum value for which the expressions (2) and (3) have a physical meaning (condition $\left.R\left(t^{\circ} \mathrm{C}\right)>0\right)$. So:

$$
R\left(t^{\circ} \mathrm{C}\right)=\lim _{m \rightarrow 0} \frac{R_{\mathrm{I}}}{1-\frac{k}{m}}=\lim _{m \rightarrow 0} \frac{R_{\mathrm{I}}}{1-1}=+\infty .
$$

Let's analyze how the change in the mass of the winding affects its active component of resistance. The active component of the resistance is determined in accordance with the expression:

$$
R=\frac{\rho_{\mathrm{M}} \cdot l_{\mathrm{M}}}{S_{\mathrm{M}}}
$$

where $\rho_{\mathrm{M}}$ - resistivity of copper; $l_{\mathrm{M}}$ - length of copper wire; $S_{\mathrm{M}}$ - cross-sectional area of a copper wire. The mass of a copper wire is determined by the expression:

$$
m=p_{\mathrm{M}} \cdot V_{\mathrm{M}}=p_{\mathrm{M}} \cdot l_{\mathrm{M}} \cdot S_{\mathrm{M}},
$$

where $V_{\mathrm{M}}$ - volume of copper wire.

From expression (6) we express the length of a copper wire:

and substitute this expression in (5):

$$
l_{\mathrm{M}}=\frac{m}{p_{\mathrm{M}} \cdot S_{\mathrm{M}}},
$$

$$
R=\frac{\rho_{\mathrm{M}}}{S_{\mathrm{M}}} \cdot \frac{m}{p_{\mathrm{M}} \cdot S_{\mathrm{M}}}=\frac{\rho_{\mathrm{M}}}{p_{\mathrm{M}}} \cdot \frac{m}{S_{\mathrm{M}}^{2}} .
$$

Let's analyze expression (7). Ratio $\frac{\rho_{\mathrm{M}}}{p_{\mathrm{M}}}$ is the ratio of the constants and does not change under the same external conditions. On the one hand, the reduction in mass leads to a decrease in the active component of the winding resistance, and on the other hand this decrease can be more than offset by an increase in the active component of the winding resistance due to the reduction in the diameter of the copper wire, because the active component of the winding resistance is inversely proportional to the second degree of the cross-sectional area.

We will consider a special case of optimizing the amount of copper used to make the working winding of an electromagnet, in which the geometry of the magnetic circuit has not changed, the number of turns is wound up, and we introduce simplification that a change in the diameter of the wire did not lead to a change in the wire length, and $l_{1}=l_{2}=l$. Then from (5), taking into account that $p_{\mathrm{M}}$ is a constant, and $I$ is not changing, it follows that in this case the mass of the copper wire of the winding is directly proportional to the area of its cross section, then in accordance with formula (6), if the winding mass is reduced by a factor of $x$, the active component of the winding resistance will only decrease by $x$, due to the reduction in the cross-sectional area, will increase by $x^{2}$ times, which ultimately leads to an increase in the active component of the winding resistance by a factor of $x$.

World trends in the reduction of the overall dimensions of EP in general and electromagnets in particular lead to the maintenance of the same performance 
characteristics for smaller mass-size parameters or to provide higher operating parameters with the same mass and size characteristics, which is achieved through the use of the magnetic capabilities of magnetic circuits, and electrical working winding of the product, in particular, a reduction in the diameter of the winding wire leads to an increase in its active component of the resistance, but allows the use of less copper, while making the same number of turns. All this leads to the fact that the weight and dimensions parameters (mass, geometric dimensions) decrease, and $R\left(t^{\circ} \mathrm{C}\right)$ grows at each stage of improving the EP. The analysis of expressions (2) and (3) shows that the trends of miniaturization lead both to a greater absolute value of the active component of the winding resistance, and to its greater increment in the process of sensorless determination of the magnetic characteristic of the EP.

Authors are invited to use the exact ACWR values obtained to accurately determine the winding temperature at any time during the EP test in accordance with the expression (obtained from expression (1) with other notations):

$$
T_{k}=T_{0}+\frac{R_{k}-R_{0}}{\alpha R_{0}}
$$

where $R_{0}$ - value of ACWR in normal conditions, $R_{k}$ - the value of the winding temperature for each $k$ measurement.

An iterative calculation can be used, then it is necessary to determine additionally the initial temperature or to calculate not the absolute value of the temperature, but to determine the temperature rise (i.e., $T_{0}=273 \mathrm{~K}$ ):

$$
T_{k}=T_{k-1}+\frac{R_{k}-R_{k-1}}{\alpha R_{k-1}} .
$$

\section{Results and discussion}

A proportional electromagnet was tested with the help of KEK-03-MMM-150 (IIP IIIS, Russia) and the necessary electrical parameters were obtained to calculate the ACCO according to expressions (2) and (3). ACWR is varies linearly, since an effective current value was used to calculate it; $R\left(t^{\circ} \mathrm{C}\right)_{k}$ varies wavy, since the change is determined by the amount of heat released, which is proportional to the square of the sinusoidal current.

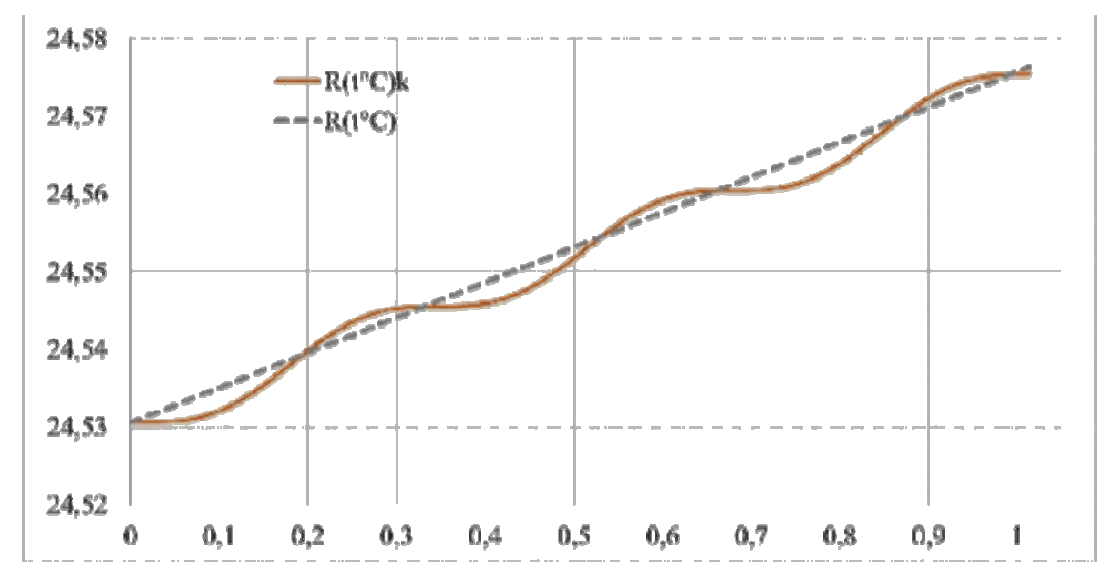

Fig. 1. Time diagrams of the calculated active component of the resistance of the EP winding. 
Figure 2 shows the time diagrams of the winding temperature excess obtained in accordance with the above expressions (8) and (9), the curves obtained are practically identical, since they differ less than $0,1 \%$.

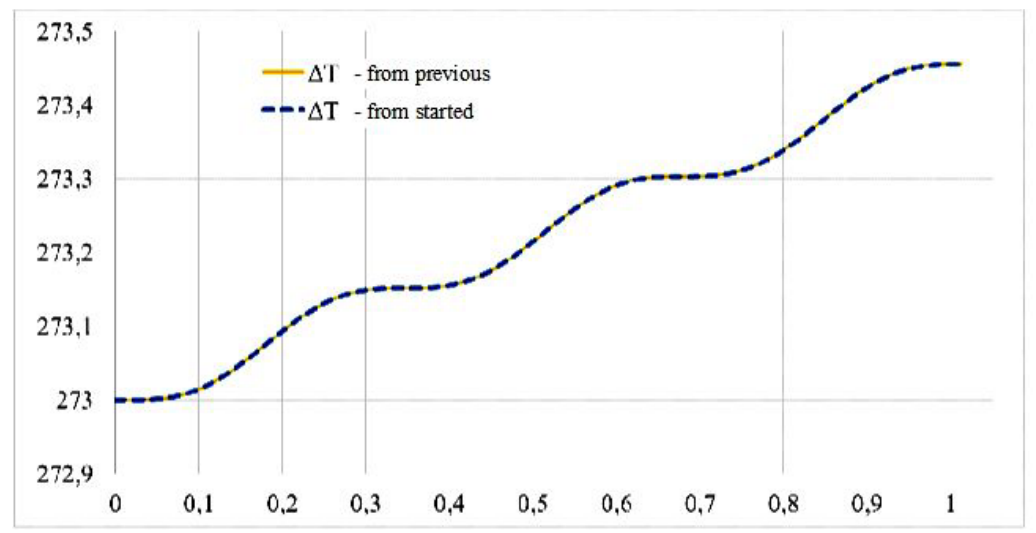

Fig. 2. Time diagrams of the calculated winding temperature EP.

Tracking the temperature of the working winding of the EP makes it possible to prevent damage to its insulation, the failure of the entire product.

\section{Conclusion}

Based on the conducted studies, a method has been developed for determining the active component of the winding resistance during the EP test, taking into account the heat energy released.

Taking into account the nature of the change in ACWR in accordance with expressions (2) and (3), and also that ACWR is growing due to the released thermal energy, a method of operational sensorless measurement of the weber-ampere characteristic of the EP has been developed. It differs from the known ones in that, in order to compensate for the incorrect definition of ACWR, the heat energy in the winding. The method provides high accuracy in determining the characteristics for short-term tests of EP is under normal conditions (in the steady state, that is, the temperature of the electromagnet corresponds to the ambient temperature). The essence of the method is as follows:

1. The active component of the winding resistance is measured directly before measuring the magnetic characteristic $-R_{\mathrm{I}}$, and after measurement $-R_{\mathrm{II}}$.

2. The change in the active component of the winding resistance is calculated: $\Delta R=R_{\mathrm{II}}-R_{\mathrm{I}}$.

3. The thermal energy extracted in the winding is calculated for each $k$-step of the measurement (for $k=1 . . N-$ the number of measured points, when determining the magnetic characteristic): $W_{k}=i_{k}^{2} \cdot R_{\mathrm{I}} \cdot\left(t_{k}-t_{k-1}\right)$, where $i_{k}, t_{k}$ - current and time values for each $k$ measurement.

4. There is total thermal energy allocated in the object when measuring the magnetic characteristic: $W_{\Sigma}=\sum_{k=1}^{N} W_{k}$. 
5. The coefficient between the correspondence between the increment of the active component of the resistance and the increment of the total thermal energy is calculated: $p=\frac{\Delta R}{W_{\Sigma}}$.

6. The ACWR increment values corresponding to each $k$ measurement step are calculated: $\Delta R_{k}=p \cdot W_{k}$.

7. The ACWR values corresponding to each $k$ measurement step are determined: $R_{k}=R_{k-1}+\Delta R_{k}$, wherein $R_{1}=R_{\mathrm{I}}$, (for $k=1$ ).

8. The flux-linkage differentials corresponding to each $k$ measurement are calculated: $d \psi_{k}=\left(u_{k}-i_{k} \cdot\left(R_{k}+R_{s h}+R_{\mathrm{pr}}\right)\right) \cdot\left(t_{k}-t_{k-1}\right)$, where $u_{k}$ - voltage values on the winding for each $k$ measurement; $R_{\mathrm{s} h}$ - shunt resistance; $R_{\mathrm{pr}}$ - resistance of connecting wires, which for small ACWR values can not be neglected. And it is assumed that $d \psi_{1}=0$.

9. The flux-linkage values are found for each $k$ dimension: $\psi_{k}=\psi_{k-1}+d \psi_{k}, \psi_{1}=0$.

10. A proportional closure of the magnetic characteristic is performed, for which the initial curve and the upper hysteresis loop are shifted downward, and the lower half of the hysteresis loop is proportionally shifted upwards until the coincidence $\psi_{m 1}$ and $\psi_{m 2}-1$ and 2 values of saturation flux coupling: $\psi_{k_{3} .}=\psi_{\min }+\left(\psi_{k}-\psi_{\min }\right) \cdot a$, where $\psi_{\min }-$ minimum value of the calculated flux linkage; $a$ - coefficient, which is defined by expression:

$$
a=\left\{\begin{array}{l}
\frac{\psi_{m 1}-\psi_{\min }-0,5 \cdot \Delta \psi_{m}}{\psi_{m 1}-\psi_{\min }}, \text { for the starting and higher parts of the hysteresis loop; } \\
\frac{\psi_{m 2}-\psi_{\min }+0,5 \cdot \Delta \psi_{m}}{\psi_{m 2}-\psi_{\min }}, \text { for the lower part of the hysteresis loop. }
\end{array}\right.
$$

where $\Delta \psi_{m}=\psi_{m 1}-\psi_{m 2}$ - the value of the non-convergence of the hysteresis loop.

11. The value of the flux link correction is calculated: $\psi_{\text {popr. }}=\frac{\psi_{\max n o n-c .}+\psi_{\min \text { non-c. }}}{2}$, where $\psi_{\max \text { non-c. }}, \psi_{\min \text { non-c. }}-$ the maximum and minimum values of the calculated noncentered flux linkage.

12. The values of the centered flux linkages corresponding to each $k$ measurement: $\psi_{k \text { cent. }}=\psi_{k \text { z. }}-\psi_{\text {popr. }}$.

13. A weber-ampere characteristic of the test sample is constructed $-\psi_{k \text { cent. }}(I)$.

The proposed method allows to measure the magnetic characteristics of EP with high accuracy, analyzing which can be diagnosed by EP, for example, analysis of web-ampere characteristics of proportional electromagnets allows detecting the following defects: interturn short circuit; clogging on the upper surface of the anchor; defect of the spring; change in the magnetic characteristics of the magnetic circuit; deep crimping of the valve; damage to the sealing element; Anchoring in the initial position; sticking anchor in a tightened position. In addition, it is important to monitor the temperature of the winding EP, exceeding a certain value of which leads to damage to insulation, the failure of the entire product. To accurately control the temperature of the use of temperature sensors is usually difficult, if they were not specifically installed in this manufacturer, which is extremely rare. Installation of external sensors allows you to determine the temperature of the upper layers of the winding or reflects the complex temperature of the winding and the parts of 
the EP contacting it. Therefore, the problem of accurately determining the temperature of the winding is an actual problem.

The reported study was funded by RFBR according to the research project № 18-38-00682 using equipment of shared facility "Diagnosis and energy-efficient electrical equipment" (NPI).

\section{References}

1. J Jiang, J. Tian, J. of Chongqing University, 38(4), 75 (2015)

2. D. Shaykhutdinov, A. Lankin, N. Narakidze, V. Grechikhin, K. Shirokov, N. Gorbatenko, Research J. of Appl. Sci., 10(10), 555 (2015)

3. A.A. Sakhovova, K.M. Shirokov, S.G. Janvarev, Mod. prob. of sci \& education, 5, 111 (2013)

4. B. Kiran Kumar, Y.V. Siva Reddy, M. Vijaya Kumar, Int. J. of Control Theory and Appl., 8(1), 71 (2015)

5. M. Zolfaghari, S.A. Taher, D.V. Munuz, Ain Shams Eng. J., 7(2), 729 (2016)

6. R.I. Leukhin, D.V. Shaykhutdinov, V.I. Leukhin, Proc. Eng., 150, 347 (2016)

7. J.-S. Choi, J.-S. Ko, D.-H. Chung, 2006 SICE-ICASE Int. Joint Conf., 690 (2016)

8. F. Meng, H. Zhang, D. Cao, H. Chen, IEEE/ASME Transactions on Mechatronics 21(3), 1742 (2016)

9. N.I. Gorbatenko, A.M. Lankin, M.V. Lankin, Proc. Eng., 150, 1027 (2016)

10. A. Zohoori, A. Vahedi, S. Meo, V. Sorrentino, J. of Intellig. and Fuzzy Syst., 30(1), 159 (2016)

11. V.V. Grechihin, M.V. Lankin, A.M. Lankin, Proc. Eng., 129, 793 (2015) 